\title{
Situation Detection in a Movement-Assistive Device
}

\author{
Jürgen Hielscher ${ }^{1}$, Andreas Horn ${ }^{2}$, Markus Grün ${ }^{2}$, Markus Hessinger ${ }^{1}$, \\ Mark Schindler ${ }^{2}$, Fabian Bauer ${ }^{1}$, Roland Werthschützky ${ }^{1}$ \\ ${ }^{1}$ Institute of Electromechanical Design, Technische Universität Darmstadt, \\ Merckstrasse 25, 64283 Darmstadt, Germany \\ j.hielscher@emk.tu-darmstadt.de \\ ${ }^{2}$ Institut für Automatisierungstechnik und Mechatronik, Technische Universität Darmstadt, \\ Landgraf-Georg Straße 4, 64283 Darmstadt, Germany
}

\begin{abstract}
The collaboration of human and robot in an intuitive and synergistic way is crucial for all movementassistive devices, such as exoskeletons and powered orthoses. This paper reports about the approach to estimate the situations of human everyday-life to control a powered knee-ankle-foot orthosis. A fuzzy-logic based algorithm evaluates the sensor signals of ground reaction forces, knee angles and muscle activity. With this setup, the seven most relevant situations for human mobility can be distinguished in $90 \%$ of the recorded time. All situation transitions were recognized correctly.
\end{abstract}

Key words: situation detection, assistive device, muscle activity, exoskeleton, fuzzy

\section{Introduction}

Technologies in the field of assistive robotics are evolving worldwide [1, 2]. Those systems help people to recover lost abilities after injury or illness, compensate weakness or to augment their skills like strength, sensitivity or accuracy.

The overarching design goal for the controller of an assistive device is the collaboration of human and robot in an intuitive and synergistic way [3]. Therefore the machine has to recognize the current situation and adapt to assist properly.

This paper focuses on the situation detection system for a powered lower limb orthosis presented in $[4,5]$. The leg-exoskeleton is designed to support the elderly in demanding movements, such as standing up from a seated position or stair climbing, by providing an external torque to the knee (Fig. 1). The overarching goal is to provide an independent life at home and beyond. The orthosis recognizes the current movement situation by evaluating the user's posture, motion and muscle activity and adapts to a suitable power support.

\section{Powered KAFO and sensing system}

The assistive device is based on a knee-anklefoot-orthosis (KAFO). The torque is provided by a series-elastic-actuator (SEA) mounted on the thigh-shells. Sensors are needed to calculate the current physiological knee torque and for situation detection. We integrated force sensors in each sole to measure the vertical groundreaction-forces (GRF). The angles of knee and ankle are captured using digital encoders in the hinge joints.

Additionally, the activity in the knee extensor muscles (musculus quadriceps femoris) is recorded. It provides information about the user's intention to start, continue or end a movement and hence can indicate the beginning of a new situation.

To derive muscle activity, electromyography (EMG) is a widely used standard [6]. However, to record the electrical activity, electrodes with skin-contact are necessary. Besides the discomfort of glued electrodes, the measurement is affected by sweat, aging effects of the interface and electromagnetic disturbances and hence not suitable for the long-term usage in an assistive device for the elderly. We developed a different approach to detect the muscle activity of the knee extensor muscles [7]. During contraction the muscle generates tension and thus a deformation of the muscle and the surrounding tissue. The cross section profile of the muscle changes during contraction from flat oval-shape towards round shape. These effects result in a bulging of the muscle and regarding the cross-sectional profile of the thigh, a higher skin surface curvature. 


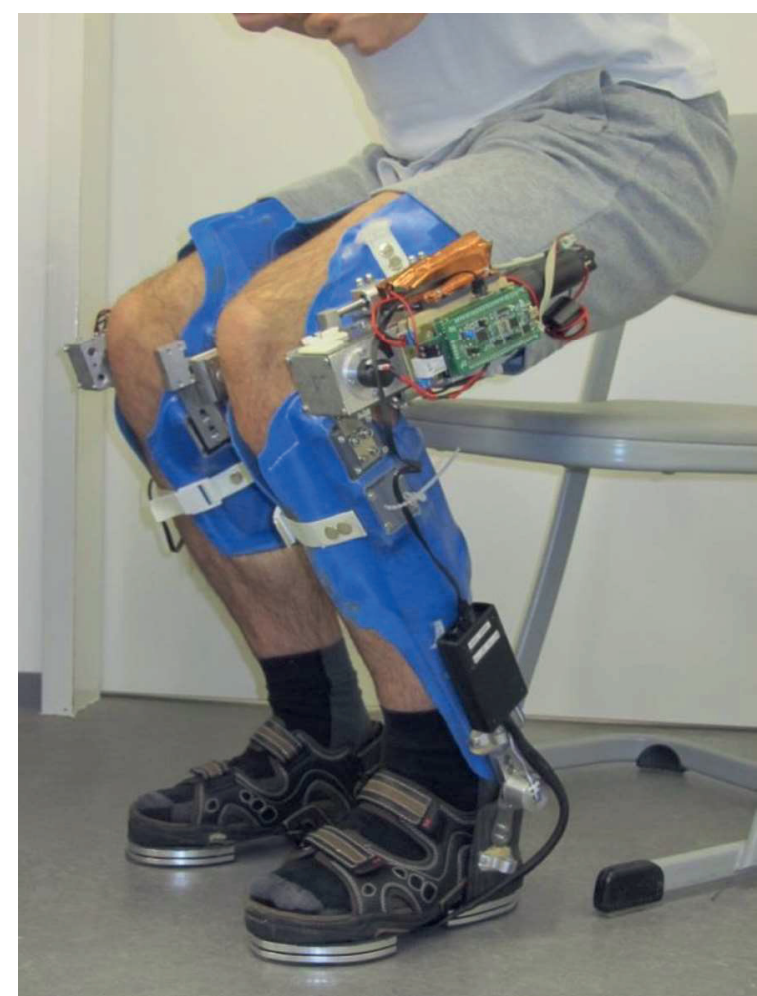

Fig. 1: User with Powered KAFO while standing up from a chair.

To convert the mechanical deformation into an electrical signal, we use a flex sensor (FSL0095103ST, Spectra Symbol, USA). It is integrated in a cuff and placed on the thigh above the muscle belly (Fig. 2). Thus, the contraction of the knee extensor muscle yields to a flexion of the sensor and hence an increase of its electrical resistance. The muscle activity sensor (MAS) can be worn for hours without affecting the signal quality, is comfortable to wear and easy to integrate in the KAFO.

From the sensor signals the following kinetic and kinematic variables are derived:

$\Sigma$ GRF: The sum of the GRF from left and right leg scaled to bodyweight

$0=$ feet unloaded

$1=$ full bodyweight on the feet

$\Delta G R F$ : The distribution of the GRF scaled to the sum of the GRF

$0=\mathrm{GRF}$ are equally distributed on both feet

$1=\mathrm{GRF}$ is shifted to one foot

$\varphi_{\mathrm{K}} \quad$ Knee angle left and right in ${ }^{\circ}$ $0^{\circ}=$ knee is fully stretched $>0^{\circ}$ knee is bent

$\Delta \varphi_{\mathrm{K}} \quad$ Absolute difference of the knee angles left and right in ${ }^{\circ}$
$\overline{\varphi_{\mathrm{K}}} \quad$ Mean of the knee angles left and right in $^{\circ}$

$\dot{\varphi_{K}} \quad$ Knee angle velocity left and right in $\%$

$\dot{\overline{\varphi_{K}}} \quad$ Mean of the knee angle velocity left and right in $\%$

MA Muscle activity of the knee extensor muscles left and right scaled to maximum strength

$\Sigma \mathrm{MA}$ Scaled sum of MA

$\Sigma \dot{\mathrm{M} A} \quad$ Scaled first derivation of $\Sigma \mathrm{MA}$

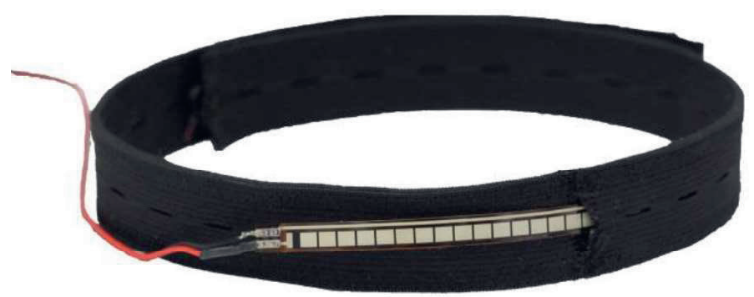

Fig. 2.:MAS: The flex sensor is integrated in an elastic cuff.

\section{Situation Detection Algorithm}

Seven situations are defined since they are considered to be most relevant for human mobility: sitting (SI), standing up from seated position (SU), standing (ST), sitting down to seated position (SDo), walking (W), stairascending (SA) and stair-descending (SDe).

Based on a set of rules, the probability of each situation is calculated from the kinetic and kinematic variables. The situation with the highest probability is assumed to be the current situation.

In a first step, the situation detection algorithm differentiates whether movement is synchronous (SI, ST, SU, SDo) or asynchronous (W, SA, SDe) regarding the legs and switches to the corresponding subsystem. The asynchronous subsystem is activated if the statement

$$
(\Delta G R F>0,8) A N D\left(\overline{\varphi_{K}}<70^{\circ}\right) A N D(\Delta G R F>0,95)
$$

becomes true. The synchronous subsystem is activated, if $\Delta G R F<0,95$ for one second or any other condition of the statement becomes false. The timer of one second is needed to catch the bipod stand phase in each asynchronous movement. 

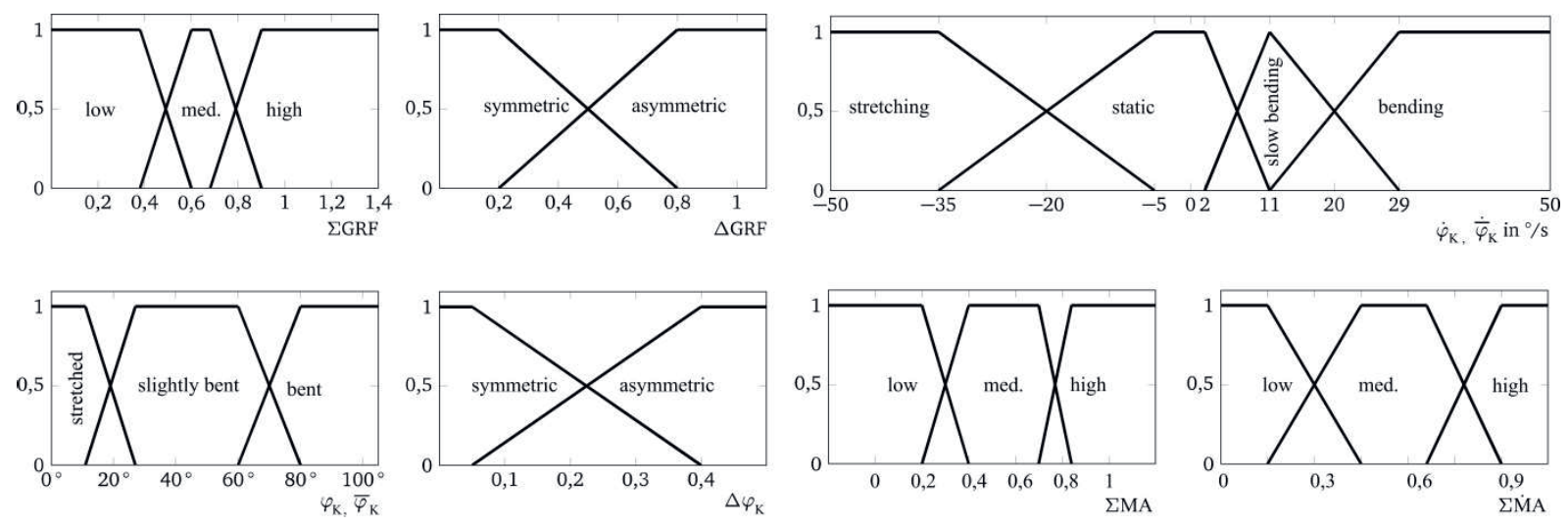

Fig.3: Fuzzy membership functions and linguistic variables. Each function maps one measured variable to a truth value between 0 and 1.

Tab.1: Fuzzy rules of the synchronous subsystem. Entries in columns are connected with the logical AND function, corresponding rows with the logical OR function. "-"represents "don't care".

\begin{tabular}{l|ccccccc} 
Situation & $\Sigma$ GRF & $\Delta$ GRF & $\overline{\varphi_{K}}$ & $\Delta \varphi_{K}$ & $\dot{\overline{\varphi_{K}}}$ & $\Sigma \mathrm{MA}$ & $\Sigma \dot{\mathrm{MA}}$ \\
\hline \hline SI & low & - & - & - & - & - & - \\
\hline SU & - & sym. & $\begin{array}{c}\text { not } \\
\text { stretched }\end{array}$ & sym. & not static & high & - \\
\hline SU & - & sym. & $\begin{array}{c}\text { not } \\
\text { stretched }\end{array}$ & sym. & not static & - & high \\
\hline SDo & - & sym. & $\begin{array}{c}\text { not } \\
\text { stretched }\end{array}$ & sym. & $\begin{array}{c}\text { slow } \\
\text { bending }\end{array}$ & - & - \\
\hline ST & high & - & stretched & sym. & - & not high & - \\
\hline
\end{tabular}

Tab.2: Fuzzy rules of the asynchronous subsystem.

\begin{tabular}{l|cccc} 
Situation & $\varphi_{K}$ right & $\varphi_{K}$ left & $\dot{\varphi}_{K}$ right & $\dot{\varphi}_{K}$ left \\
\hline $\mathrm{W}$ & stretched & stretched & - & - \\
\hline $\mathrm{W}$ & - & - & bending & - \\
\hline $\mathrm{W}$ & - & - & - & bending \\
\hline $\mathrm{SA}$ & $\begin{array}{c}\text { slightly } \\
\text { bent }\end{array}$ & stretched & - & - \\
\hline $\mathrm{SA}$ & stretched & $\begin{array}{c}\text { slightly } \\
\text { bent }\end{array}$ & - & - \\
\hline $\mathrm{SDe}$ & stretched & $\begin{array}{c}\text { slightly } \\
\text { bent }\end{array}$ & - & - \\
\hline SDe & slightly & stretched & - & - \\
\hline
\end{tabular}


The situation is determined in the currently active subsystem by evaluating the user's posture and motion. The individual posture is indistinct. The statement "my leg is stretched" is surely true for $\varphi_{K}=0^{\circ}$. But it is still somewhat true for $\varphi_{K}=20^{\circ}$. Therefore we chose a fuzzy logic based approach. All variables are mapped into fuzzy membership functions (truth values). In this approach we used ramp functions only (Fig. 3). The fuzzy rules connect two or more truth values in order to calculate the truth value of each situation. The definition of the fuzzy rules is crucial to achieve a distinct result. All fuzzy rules used in our situation detection algorithm are listed in Table 1 and 2. To calculate the truth value of each situation the most common approach was chosen: the minimum-function resolves the logical $\mathrm{OR}$ and the maximum-function resolves the logical AND. The decision for one defuzzified situation is based on the highest truth value.

Two additional actions increase the reliability of the decision. In asynchronous mode, a transition of the situation is only allowed when a step is recognized. The algorithm awaits the next step and then decides about the current situation. Additionally, if a transition is detected, the logical situation succession is verified. For example, SA cannot follow directly after SI (Fig. 4). If a forbidden transition is detected, the last situation is kept.

\section{Reference signal and tests}

The characteristics of a properly working situation detection algorithm are:

1. The detected situation is correct

2. The transition is detected with negligible delay

To assess the situation detection algorithm in a practical trial, a reference signal is necessary. Therefore, additional input signals are used. Via pushbutton the user indicates the beginning and completion of each movement. In combination with a predefined movement sequence, the situations can be distinguished in the recorded data. In order to determine the instant of the situation transition, two additional sensors are used. A chair is equipped with a force sensor to detect the seat-on and seat-off, i.e. the instant when the user touches the chair during SDo and loses contact during SU respectively. The electromyogram of the knee extensor muscles is recorded in addition to the MAS, since it represents the earliest possible signal to indicate the beginning of a movement.
All signals were recorded simultaneously at $1080 \mathrm{~Hz}$. The signals from the MAS are lowpass filtered $(20 \mathrm{~Hz})$, the derived signal $\Sigma \dot{\mathrm{M} A}$ is filtered once again $(20 \mathrm{~Hz})$.

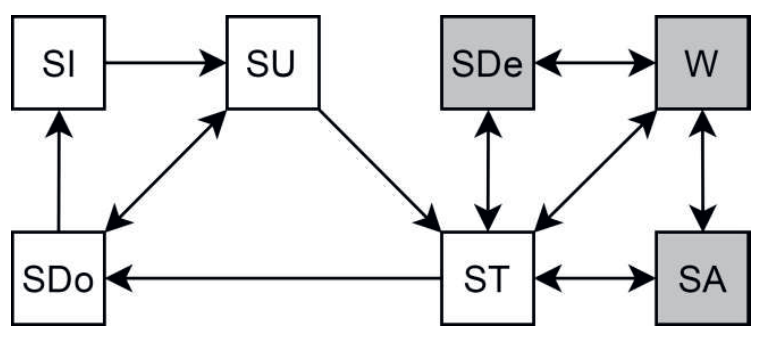

Fig. 4. Synchronous (white boxes) and asynchronous (grey boxes) situations and valid transitions.

The situation detection algorithm was tested on one healthy subject (male, age 24 , weight $77 \mathrm{~kg}$, height $1,84 \mathrm{~m}$ ). The subject was equipped with the KAFO and the additional sensors for the reference. The KAFO was operated passive, i.e. without power support. All seven situations were performed in a predefined sequence.

\section{Results and Discussion}

The algorithm detects the correct situation in $90 \%$ of the recorded time. All transitions were recognized correctly. The amount of time in which the algorithm's decision deviates from the reference is due to delay only.

The delay is mostly caused by the timer between the synchronous and the asynchronous subsystem. The algorithm waits for one second until the situation ST can be distinguished from the bipod stand phase.

The most important situation is SU, since it requires the highest power on the knee. The transition from $\mathrm{SI}$ to $\mathrm{SU}$ is recognized even before the seat-off, which helps to gain trust in the assistive device.

\section{References}

[1] R. Bogue, "Robotic exoskeletons: a review of recent progress," Ind. Robot An Int. J., vol. 42, no. 1, pp. 5-10, 2015.

[2] R. Bogue, "Exoskeletons and robotic prosthetics: a review of recent developments," Ind. Robot An Int. J., vol. 36, no. 5, pp. 421-427, 2009.

[3] M. R. Tucker, J. Olivier, A. Pagel, H. Bleuler, M. Bouri, O. Lambercy, J. D. R. Millán, R. Riener, H. Vallery, and R. Gassert, "Control strategies for active lower extremity prosthetics and 
orthotics: a review.," J. Neuroeng.

Rehabil., vol. 12, no. August 2016, p. 1, 2015.

[4] J. Hielscher, R. Müller, T. Meiss, A. Horn, J. Block, S. van Drongelen, U. Konigorski, H. F. Schlaak, P. P. Pott, and R. Werthschützky, "Powered Active Knee-Ankle-Foot-Orthosis," in BMT 2015 - 49th DGBMT Annual Conference, 2015.

[5] P. Pott, S. Wolf, J. Block, S. Van Drongelen, M. Grün, D. Heitzmann, J. Hielscher, A. Horn, R. Müller, O. Rettig, U. Konigorski, R. Werthschützky, H. F. Schlaak, and T. Meiss, "Knee - Ankle Foot Orthosis with Powered Knee for Support in the Elderly," J. Eng. Med., pp. 1-28, 2016.

[6] G. Robertson, G. Caldwell, J. Hamill, G. Kamen, and W. Saunders, Research Methods in Biomechanics. Human Kinetics, 2013.

[7] J. Hielscher, T. Meiss, J. Block, and R. Wertschützky, "Estimating Muscular Activity of the Quadriceps Femoris Muscles by Measuring the Change in Shape," Sens. 2013, pp. 638-641, 2013. 